\title{
SHOCKS AND A GIANT PLANET IN THE DISK ORBITING BP PISCIUM?
}

\author{
C. Melis ${ }^{1,5}$, C. Gielen ${ }^{2,6}$, C. H. Chen ${ }^{3}$, Joseph H. RheE ${ }^{4}$, Inseok Song ${ }^{4}$, And B. Zuckerman ${ }^{1}$ \\ ${ }^{1}$ Department of Physics and Astronomy, University of California, Los Angeles, CA 90095-1547, USA; cmelis@ ucsd.edu \\ ${ }^{2}$ Instituut voor Sterrenkunde, Katholieke Universiteit Leuven, Celestijnenlaan 200D, 3001 Leuven, Belgium \\ ${ }^{3}$ Space Telescope Science Institute, 3700 San Martin Dr., Baltimore, MD 21218, USA \\ ${ }^{4}$ Department of Physics and Astronomy, University of Georgia, Athens, GA 30602-2451, USA \\ Received 2010 January 14; accepted 2010 August 6; published 2010 November 3
}

\begin{abstract}
Spitzer Infrared Spectrograph data support the interpretation that BP Piscium, a gas and dust enshrouded star residing at high Galactic latitude, is a first-ascent giant rather than a classical T Tauri star. Our analysis suggests that BP Piscium's spectral energy distribution can be modeled as a disk with a gap that is opened by a giant planet. Modeling the rich mid-infrared emission line spectrum indicates that the solid-state emitting grains orbiting BP Piscium are primarily composed of $\sim 75 \mathrm{~K}$ crystalline, magnesium-rich olivine; $\sim 75 \mathrm{~K}$ crystalline, magnesium-rich pyroxene; $\sim 200 \mathrm{~K}$ amorphous, magnesium-rich pyroxene; and $\sim 200 \mathrm{~K}$ annealed silica (cristobalite). These dust grains are all sub-micron sized. The giant planet and gap model also naturally explains the location and mineralogy of the small dust grains in the disk. Disk shocks that result from disk-planet interaction generate the highly crystalline dust which is subsequently blown out of the disk mid-plane and into the disk atmosphere.
\end{abstract}

Key words: accretion, accretion disks - circumstellar matter - infrared: stars - planet-disk interactions - stars: individual (BP Piscium)

Online-only material: color figures

\section{INTRODUCTION}

BP Piscium (BP Psc) is an enigmatic high Galactic latitude star enshrouded in gaseous and dusty material (Zuckerman et al. 2008, hereafter Z08). Initially, BP Psc was thought to be an isolated K0-type star undergoing its classical T Tauri star phase. Observations performed by Z08 instead found several indications that BP Psc is likely a first-ascent giant star that accretes material from a surrounding disk of gas and dust while launching bipolar jets. The first such sign that BP Psc might not be young was a lithium $\lambda 6708$ absorption line equivalent width that was $\sim 7$ times less than what would be expected for an early K-type classical T Tauri star. No known example exists in the literature of such strong lithium depletion for a solar-like premain sequence star. A second indication that BP Psc is not young came from a low measured surface gravity. Although young stars typically have lower surface gravity than main sequence stars (as they are still contracting toward the main sequence), BP Psc's measured gravity of $10^{2.5} \mathrm{~cm} \mathrm{~s}^{-2}$ is significantly lower than those expected for 1-10 Myr old stars (which have gravity values $\gtrsim 10^{3.5} \mathrm{~cm} \mathrm{~s}^{-2}$; see Baraffe et al. 1998) and only served to further exacerbate the lithium situation. Yet another indication, although weaker than the first two, was a discrepancy between model and kinematically measured masses of BP Psc in the case that it was a classical T Tauri star. Such problems disappear if instead it is assumed that BP Psc is a first-ascent giant star.

Although the sum of the observations performed by Z08 pointed toward BP Psc as an evolved rather than a young star, they were not necessarily conclusive and further investigation of BP Psc was warranted. Kastner et al. (2008) performed molecular line observations of BP Psc showing that its molecular gas characteristics are compatible with those found in the expanding envelopes of yellow supergiant stars while Kastner et al. (2010) imaged BP Psc with the Chandra X-ray Observatory finding

\footnotetext{
5 Current address: Center for Astrophysics and Space Sciences, University of California, San Diego, CA 92093-0424, USA.

6 Postdoctoral Fellow of the Fund for Scientific Research, Flanders.
}

a weak source with characteristics compatible with rapidly rotating G-type giant stars. Melis et al. (2009) reported the first unambiguous case of a dusty, accreting first-ascent giant star (TYC 4144329 2), hinting that BP Psc could be part of a previously unidentified class of disk-bearing, accreting first-ascent giant stars (Melis 2009). Here we present Spitzer Infrared Spectrograph (IRS) measurements of BP Psc. These observations were designed to identify the constituents of BP Psc's dusty disk with the hope that such additional information would clarify the evolutionary status of BP Psc and, should BP Psc indeed be a first-ascent giant star, provide clues as to how it may have been rebirthed into characteristics typically associated with young, planet-forming stars.

\section{OBSERVATIONS}

BP Psc was observed with the Spitzer Space Telescope IRS (Werner et al. 2004; Houck et al. 2004) in stare mode on 2007 August 2 with the low-resolution modules affording resolving powers of $\sim 60-120$. Since BP Psc is such an infrared bright target, one cycle of $6 \mathrm{~s}$ ramp duration for each of the two nods was sufficient to obtain well-exposed ( $\mathrm{S} / \mathrm{N} \gtrsim 100$ per pixel) spectra for each of the short-low (SL) and long-low (LL) modules. Data were pre-processed with Spitzer Science Center IRS Pipeline Version S16.1.0 for the SL modules and S17.2.0 for the LL modules. Subsequent bad pixel masking and interpolation was performed using IRSCLEAN V1. 9 and a "master" bad pixel map. The master bad pixel map was generated by running the interactive IRSCLEAN_MASK code on each of the four unique basic calibrated data spectral images per module, then combining these four output files and the campaign rogue mask that identifies known bad pixels. The purpose of the master bad pixel map method was to identify and remove bad pixels that might otherwise go unnoticed under the well-exposed spectrum.

Individual nods from a pair were subtracted, then these background-subtracted spectra were extracted and fluxcalibrated using the SPICE software package. Each extracted, 

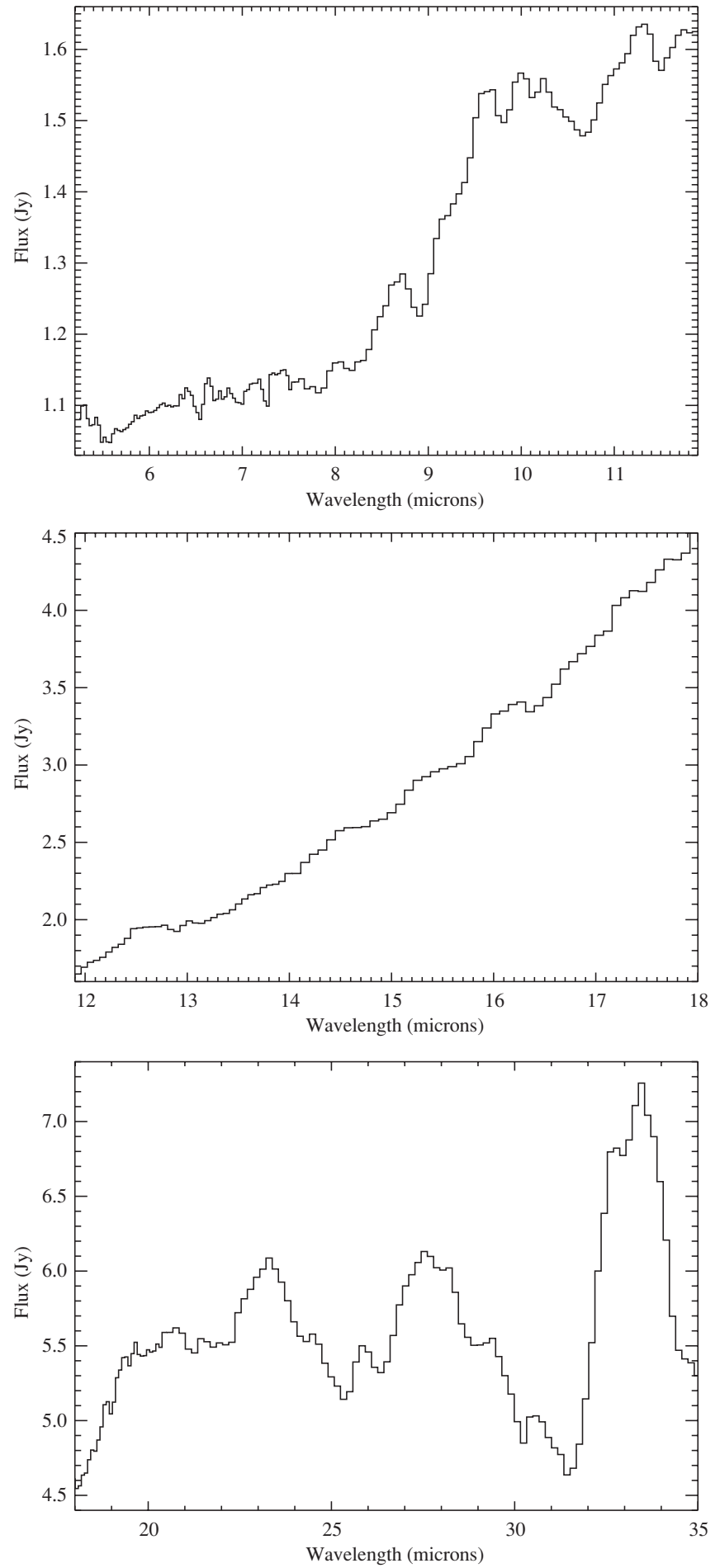

Figure 1. All panels: reduced, extracted, and calibrated IRS spectrum of BP Psc. The spectrum S/N per pixel is $\gtrsim 100$. Top panel: the $\sim 5-12 \mu \mathrm{m}$ wavelength range. Weak features in the $\sim 7-8 \mu \mathrm{m}$ range are dubious as this is an orderoverlap region. Note, however, that the $\sim 6.5 \mu \mathrm{m}$ absorption/emission complex is real and is confirmed in the spectra of both nods. Middle panel: the $\sim 12-18 \mu \mathrm{m}$ wavelength range. Weak features in the $\sim 14-15.2 \mu \mathrm{m}$ range are dubious as this is an order-overlap region. Bottom panel: the $\sim 18-35 \mu \mathrm{m}$ wavelength range. Weak features in the $\sim 19-20 \mu \mathrm{m}$ range are dubious as this is an order-overlap region. Data beyond $35 \mu \mathrm{m}$ are excluded due to the numerous bad pixels that reside in that region of the LL chip.

calibrated nod spectrum was compared against its nod pair to ensure fidelity in the final spectrum. Data from SL order 3 (the socalled bonus order) were removed due to a poor match between the two nods. Nod-averaged spectral orders were generally well matched in terms of flux continuity. Minor adjustments, where necessary, were made such that an individual order matched the flux of the bluer order. The final spectrum is displayed in detail in Figure 1 and is overplotted on a complete spectral energy distribution (SED) for BP Psc in Figure 2.

On 2007 July 8 BP Psc was serendipitously imaged at $24 \mu \mathrm{m}$ with the Multiband Imaging Photometer for Spitzer (MIPS; Rieke et al. 2004) in a program to monitor the dust cloud following Earth's orbit (AOR 18701312, PI Jayaraman; BP Psc did not fall into the $70 \mu \mathrm{m}$ field of this program). We performed aperture photometry on the $24 \mu \mathrm{m}$ post-basic calibrated data mosaic image produced by the Spitzer Science Center MIPS pipeline (version S16.1.0). We used the Spitzer Science Center recommended aperture correction of 1.167 for an aperture radius of $13^{\prime \prime}$ and sky annulus inner and outer radii of $20^{\prime \prime}$ and $32^{\prime \prime}$, respectively. We detect a flux density of $5.7 \mathrm{Jy}$ at $24 \mu \mathrm{m}$ with an uncertainty of $10 \%$, consistent with the IRAS $25 \mu \mathrm{m}$ detected flux and the IRS flux (see Figure 2).

\section{IRS MODELING}

Before discussing in detail IRS spectroscopy modeling and results, it is necessary to recall what is known about BP Psc's disk (see Z08 for details). Resolved molecular gas emission detected through Submillimeter Array aperture synthesis imaging indicates that BP Psc has gas in a disk with Keplerian orbits. Keck II adaptive optics imaging resolved a dust disk in scattered light. Near- to far-infrared photometric measurements of BP Psc reveal that $\sim 75 \%$ of BP Psc's observed light is reprocessed into the infrared and that the orbiting material is well modeled as residing in two regions having temperatures of $\sim 1500$ and $\sim 210 \mathrm{~K}$ (see Figure 2). From a Submillimeter Array continuum detection, $\mathrm{Z} 08$ calculate that $7 M_{\oplus}$ of dust orbits BP Psc in the case that it is a giant star 300 pc distant from Earth. Synthesis of the imaging data led Z08 to suggest that we view BP Psc's disk close to edge-on $\left(i \sim 75^{\circ}\right)$ and that the disk has a flared morphology.

\subsection{Disk Structure}

Acke et al. (2009) and Furlan et al. (2006) validated the use of IRS-based spectral indices to study disk structure by comparing indices computed from data to those computed from disk models. We seek to gain insight into BP Psc's disk structure by comparing its IRS spectral indices to those computed for the disks studied by Acke et al. (2009) and Furlan et al. (2006). We calculate the value of the following indices: an excess at $7 \mu \mathrm{m}$ of $4.55 \mathrm{mag}$, the ratio of flux at $13.5 \mu \mathrm{m}$ to that at $7 \mu \mathrm{m}$ ([13.5/7]) of $0.69 \mathrm{mag}$, and the ratio of flux at $30 \mu \mathrm{m}$ to that at $13.5 \mu \mathrm{m}$ ([30/13.5]) of $0.96 \mathrm{mag}$ (see Acke et al. 2009 for how these values are derived); the ratio of integrated continuum flux near $6 \mu \mathrm{m}$ to that near $13 \mu \mathrm{m}\left(n_{6-13}\right)$ of 2.10 , the ratio of integrated continuum flux near $13 \mu \mathrm{m}$ to that near $25 \mu \mathrm{m}$ $\left(n_{13-25}\right)$ of 1.93 , and the ratio of integrated continuum flux near $6 \mu \mathrm{m}$ to that near $25 \mu \mathrm{m}\left(n_{6-25}\right)$ of 2.03 (see Furlan et al. 2006 for how these values are derived). These values suggest that BP Psc has strong near-infrared excess emission and steeply rising flux toward longer wavelengths (consistent with BP Psc's SED; see Figure 2 herein and Figure 6 of Z08). As mentioned above, the real power of these indices to constrain the structure of BP Psc's disk comes from comparing them to indices derived for other well-modeled disk-bearing objects. Such comparisons are presented in Section 4. 

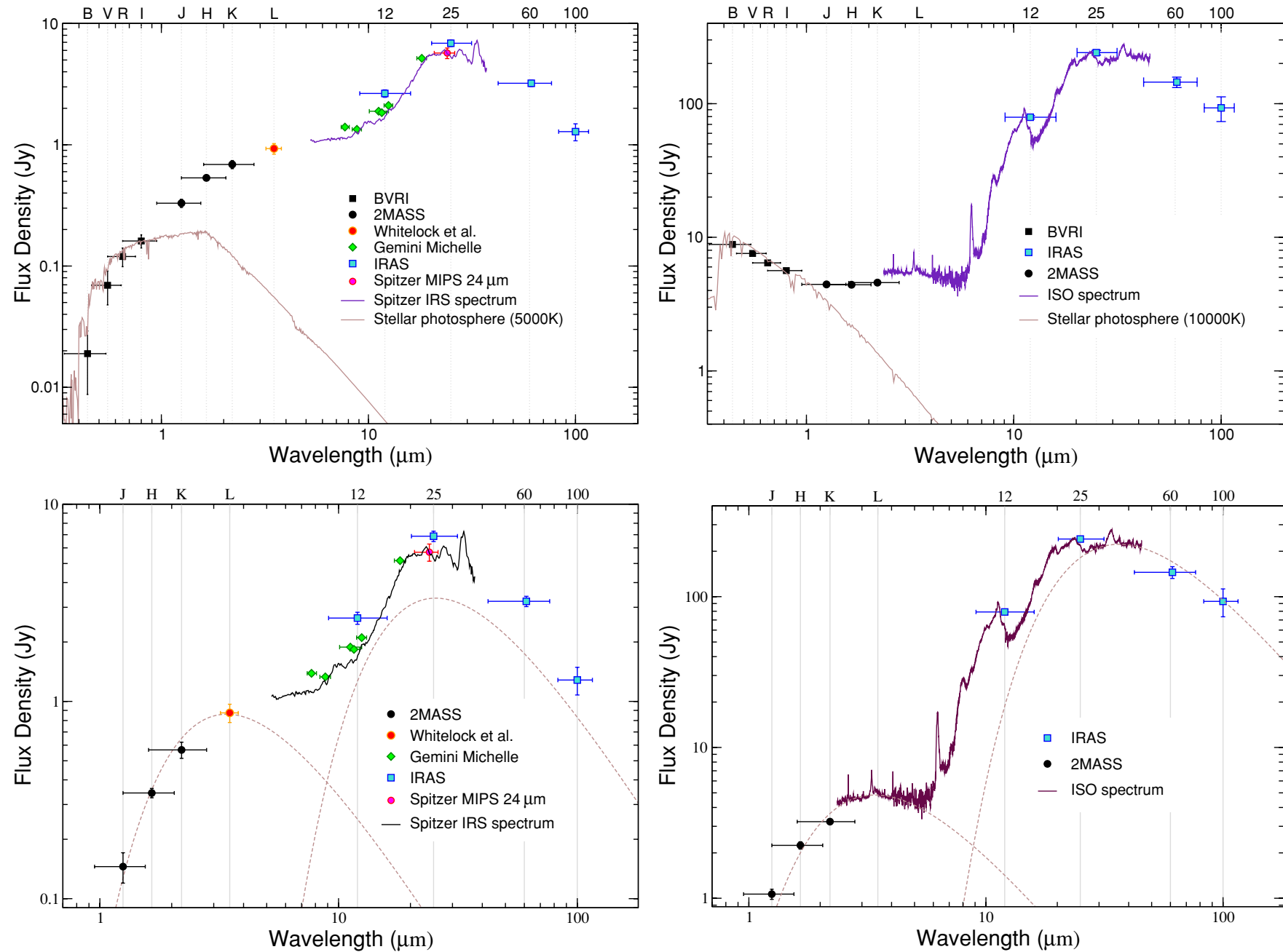

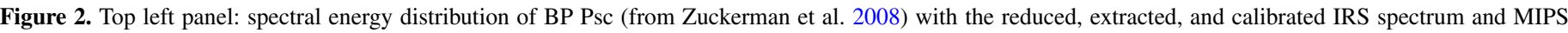

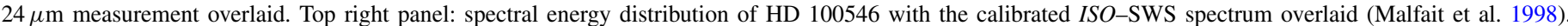

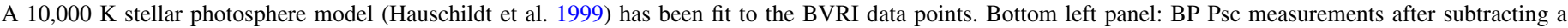

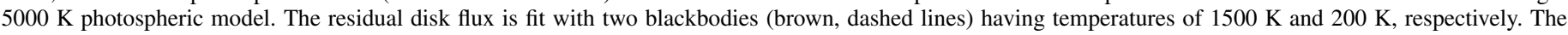

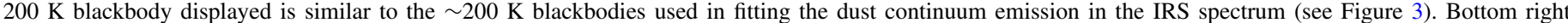

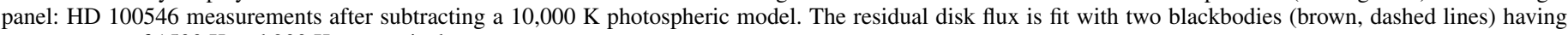
temperatures of $1500 \mathrm{~K}$ and $200 \mathrm{~K}$, respectively.

(A color version of this figure is available in the online journal.)

\subsection{Dust Composition}

The IRS spectrum of BP Psc shows several broad emission lines from solid-state transitions (Figures 1-3). To model these features, and hence deduce the nature of the emitting particles, we proceed in fitting the spectrum via two paths. In one path, we attempt to identify grain species by subtracting the most obvious contributor and searching for residuals indicative of additional grain species (as was done successfully for HD 100546 by Malfait et al. 1998). During these subtraction fits we try various grain shapes and sizes (using the absorption coefficients of Min et al. 2007) to identify the best shape distribution and grain size to use. In parallel with these subtraction fits, we fit the data using the $\chi^{2}$ minimization routine described in Gielen et al. (2008). We refer the interested reader to their work, and references cited therein, for details about model inputs. The strategy employed by Gielen et al. (2008) assumes that solid-state emission features originate in an optically thin disk atmosphere. They attempt to fit mid-infrared spectra with a blackbody model for the disk continuum emission and an emission feature model computed from the linear combination of dust absorption coefficients multiplied by different blackbody temperatures. The $\chi^{2}$ of the model fit to the data is then minimized by adjusting various parameters; e.g., grain temperature, grain species, and intensity of grain emission. Both the preliminary $\chi^{2}$ fit and subtraction fits indicate that the grains producing the emission features in BP Psc's spectrum are sub-micron $(\sim 0.1-1.0 \mu \mathrm{m})$ in size. We also find that both methods obtain consistent results in grain species identified. Similar to what was found by Gielen et al. (2008), we find that dust emission features cannot be reproduced with spherical grains (e.g., Mie theory), but are instead best reproduced with absorption coefficients calculated using the Gaussian random-field (GRF) theory for the grain shape distribution (Shkuratov \& Grynko 2005; Min et al. 2007, and references therein) and the discrete dipole approximation (Draine 1988). It is noted that the grain shape approximations used sometimes do not reproduce exactly the grain emission features (and hence likely are not a complete match to the true 


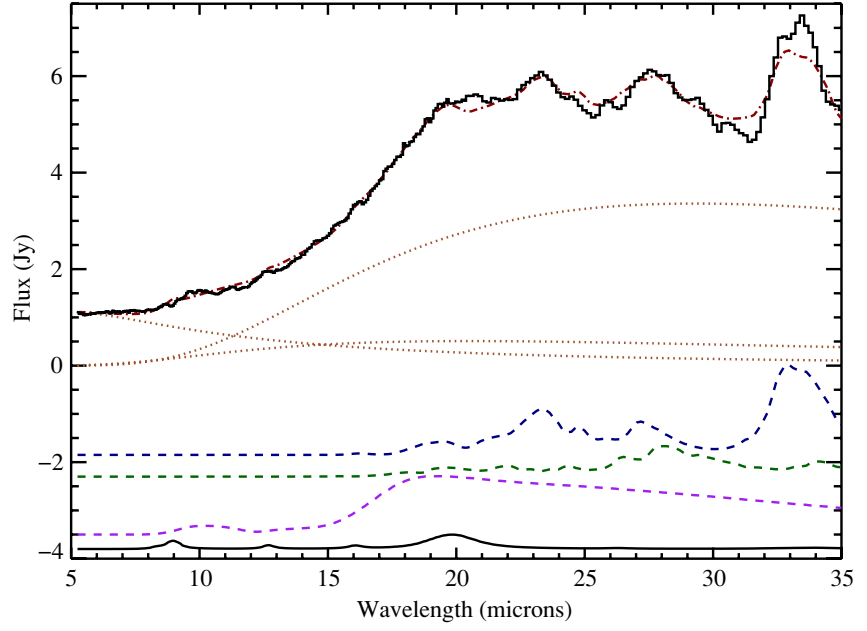

Figure 3. IRS spectrum of BP Psc; the spectrum S/N per pixel is $\gtrsim 100$. Model fits to the data (upper black curve) are overplotted with individual dust species' emissivities offset by negative flux for clarity. From bottom to top the models are for: cristobalite (black solid curve), pyroxene (purple dashed curve), enstatite (green dashed curve), forsterite (blue dashed curve), blackbodies (three brown dotted curves) having temperatures of 175,250 , and $1000 \mathrm{~K}$, respectively, and the sum of all the aforementioned curves (red dot-dashed curve). See Table 1 for the dust parameters.

(A color version of this figure is available in the online journal.)

grain shapes); see the discussion in Gielen et al. (2008). In the following three paragraphs we describe the resulting grain species identified from these two methods.

Three features-with wavelengths near 33, 28, and $23 \mu \mathrm{m}$ - stand out in BP Psc's IRS spectrum. These features are typically associated with crystalline, magnesium-rich olivine grains (forsterite, $\mathrm{Mg}_{2} \mathrm{SiO}_{4}$ ). Fits to the forsterite features leave significant residual emission in the $20-35 \mu \mathrm{m}$ range. Experimentation with various grain species indicates a contribution from crystalline, magnesium-rich pyroxene (hereafter enstatite, $\mathrm{MgSiO}_{3}$ ). Fits including enstatite leave little residual solid-state emission in the $23-35 \mu \mathrm{m}$ range.

Our forsterite and enstatite fits alone cannot reproduce the feature near $10 \mu \mathrm{m}$. The $\sim 10 \mu \mathrm{m}$ feature is typically associated with amorphous dust of the pyroxene or olivine species. Experimentation with the two suggests that amorphous, magnesiumrich pyroxene (hereafter pyroxene, $\mathrm{MgSiO}_{3}$ ) provides a better fit.

The remaining residual emission-near wavelengths of 9, 13,16 , and $21 \mu \mathrm{m}$ - is reminiscent of the features produced by annealed, crystalline silica (hereafter cristobalite, $\mathrm{SiO}_{2}$; see Sargent et al. 2009b and references therein). It is noted that the 9 and 16 micron complexes are marginally detected in the residual spectrum. In light of this fact, and in the interest of completeness, we reviewed other polymorphs of silica to see if they could reproduce the remaining spectral features. $\alpha$-quartz is ruled out by its characteristic $25 \mu \mathrm{m}$ feature which would be quite strong if present (Gervais \& Piriou 1975). $\beta$-quartz is ruled out by the high temperatures necessary to excite its resonances $\left(T_{\text {dust }} \gtrsim\right.$ $850 \mathrm{~K}$; Gervais \& Piriou 1975); our above analysis suggests the small dust grains seen in BP Psc's IRS spectrum are significantly cooler (see also below). The polymorphs coesite and stishovite are also ruled out due to their unique emission spectra (see Sargent et al. 2009b and references therein). This leaves cristobalite, amorphous silica, and obsidian as potential silica polymorphs to explain the residual emission. Since obsidian and amorphous silica have similar spectral features (see Figure 3 of Sargent et al. 2009b), we use amorphous silica to represent both polymorphs. To model silica emission features we adopt optical constants from Min et al. (2007) for amorphous silica (hereafter silica) and from C. Lisse (2009, private communication; see the discussion in the supplementary online material to Lisse et al. 2006 for a description of the input optical constants) for cristobalite. Absorption coefficients are calculated for silica using GRF and for cristobalite using a continuous distribution of ellipsoids (CDE). Models using silica result in higher $\chi^{2}$ than do fits using cristobalite and fits without any $\mathrm{SiO}_{2}$ polymorph provide yet even higher $\chi^{2}$ values; a comparison of fits using cristobalite, silica, and neither cristobalite nor silica are shown in Figure 4. As such, we consider $\mathrm{SiO}_{2}$ to be significantly detected in the IRS spectrum of BP Psc and conclude that annealed silica is the most likely polymorph.

Some of the above mentioned transitions are coincident with those of polycyclic aromatic hydrocarbons (PAHs). PAHs, however, typically display strong $8.0 \mu \mathrm{m}$ features (see, e.g., Sloan et al. 2007 and references therein). No such feature is seen in our spectrum of BP Psc, thus we conclude that PAHs do not significantly contribute to the strength of the observed solid-state features.

We briefly mention the effect of reddening on mid-infrared solid-state emission features. Highly reddened stars can exhibit depressed $10 \mu \mathrm{m}$ features (Gielen et al. 2008). BP Psc, at a Galactic latitude of $-57^{\circ} .2$, is unlikely to be experiencing any significant interstellar extinction regardless of its distance. The dusty material in orbit around this star could in theory provide substantial reddening if the grains are small. In their analysis, Z08 compared low-resolution optical spectra of BP Psc to nondusty stars of similar spectral types and showed that BP Psc is not reddened along our line of sight. Thus, reddening is unlikely to contribute to the observed strength of the $10 \mu \mathrm{m}$ structure.

With rough grain parameters identified we proceed in a final $\chi^{2}$ minimization fit to the spectrum. Input dust species, grain shapes, and grain sizes are restricted to those as determined from the subtraction and initial $\chi^{2}$ fits. We allow as free parameters three independent dust continuum temperatures, three independent dust species temperatures, two dust grain sizes $(\sim 0.1-1.0 \mu \mathrm{m}$ and $\sim 2.0-4.0 \mu \mathrm{m})$, and five dust speciesforsterite, enstatite, pyroxene, amorphous olivine, and cristobalite. The final $\chi^{2}$ minimization routine employed herein differs slightly from that used by Gielen et al. (2008). Our routine assigns grain species into one of three distinct groups where each group is allowed its own dust temperature and temperature step size in the model grid. Amorphous materials (pyroxene and olivine) form one group which has a temperature grid step of $25 \mathrm{~K}$, crystalline olivine and pyroxene form another group which has a grid step of $25 \mathrm{~K}$, and silica is in its own group that has step size of $50 \mathrm{~K}$. The final model fit is displayed in Figure 3. Temperatures, mass fractions, associated errors, and an estimated mass per species are reported in Table 1. Errors on these parameters are calculated as in Gielen et al. (2008): noise is added to each pixel with a value randomly chosen from within $\pm 1 \sigma$ of a Gaussian with fullwidth at half-maximum determined from the spectrum statistical uncertainty per pixel. In this manner 100 synthetic spectra are generated, all consistent with the data, on which we perform the same fitting procedure. The resulting slightly different fit parameters are used to derive the mean (the best-fit value) and the standard deviations. The uncertainty on the mass absorption coefficients is not taken into account in this error determination. 

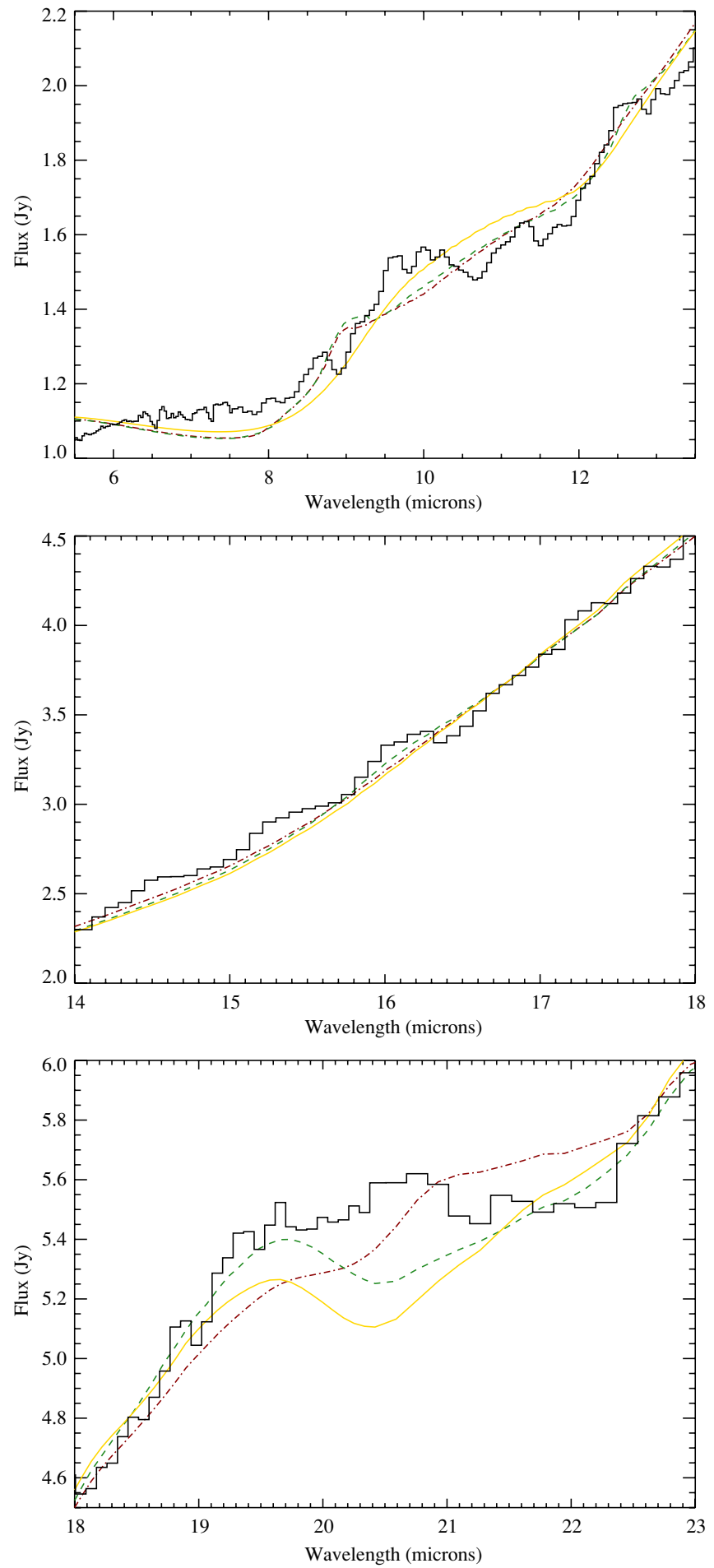

Figure 4. Regions of BP Psc's IRS spectrum having features from $\mathrm{SiO}_{2}$; the spectrum $\mathrm{S} / \mathrm{N}$ per pixel is $\gtrsim 100$. Overplotted are final dust models using cristobalite (green, dashed line; see also Figure 3), silica (red, dash-dotted line-see Section 3.2), and a model with no silica or cristobalite (gold, solid line). Fits including cristobalite result in lower $\chi^{2}$ values than those employing silica alone and neither silica nor cristobalite. Cristobalite performs better around $12.7 \mu \mathrm{m}, 16 \mu \mathrm{m}$, and $20 \mu \mathrm{m}$.

(A color version of this figure is available in the online journal.)

Here we summarize some of the main results of the spectral fitting. No larger sized $(\sim 2.0-4.0 \mu \mathrm{m})$ grains are required in the fit. Amorphous olivine $\left(\mathrm{Mg}_{2} \mathrm{SiO}_{4}\right.$, hereafter olivine $)$ is not necessary to obtain the minimum $\chi^{2}$ model fit. $\mathrm{SiO}_{2}$, however,
Table 1

Dust Component Parameters

\begin{tabular}{|c|c|c|c|c|}
\hline Dust Component & Stoichiometry & $\begin{array}{c}\text { Temperature } \\
\text { (K) }\end{array}$ & $\begin{array}{c}\text { Mass Fraction }{ }^{\mathrm{a}} \\
(\%)\end{array}$ & $\begin{array}{c}\text { Mass }^{\mathrm{b}} \\
\text { (Lunar Masses) }\end{array}$ \\
\hline Forsterite & $\mathrm{Mg}_{2} \mathrm{SiO}_{4}{ }^{\mathrm{c}}$ & $75 \pm 25$ & $59.4_{-2.4}^{+2.6}$ & 1.2 \\
\hline Enstatite & $\mathrm{MgSiO}_{3}{ }^{\mathrm{c}}$ & $75 \pm 25$ & $36.2_{-2.8}^{+2.4}$ & 0.6 \\
\hline Olivine & $\mathrm{Mg}_{2} \mathrm{SiO}_{4}{ }^{\mathrm{d}}$ & $150 \pm 25$ & $0.06_{-0.06}^{+0.35}$ & $-{ }^{\mathrm{e}}$ \\
\hline Pyroxene & $\mathrm{MgSiO}_{3}{ }^{\mathrm{d}}$ & $150 \pm 25$ & $4.0_{-0.5}^{+0.3}$ & $6 \times 10^{-2}$ \\
\hline Cristobalite & $\mathrm{SiO}_{2}{ }^{\mathrm{c}}$ & $250 \pm 50$ & $0.03_{-0.01}^{+0.06}$ & $6 \times 10^{-4}$ \\
\hline Continuum 1 & $\ldots$ & $1000_{-100}^{+50}$ & $\ldots$ & $600^{\mathrm{f}}$ \\
\hline Continuum 2 & $\cdots$ & $250_{-35}^{+25}$ & $\ldots$ & \\
\hline Continuum 3 & $\cdots$ & $175_{-30}^{+25}$ & $\cdots$ & \\
\hline
\end{tabular}

Notes.

${ }^{\text {a }}$ Fraction of mass in $0.1-1.0 \mu \mathrm{m}$, solid-state emitting grains detected in the IRS spectrum only. The reported percentage does not include the mass contained in the material responsible for the continuum emission.

${ }^{\mathrm{b}}$ Assuming the mass of the moon is $7.35 \times 10^{25} \mathrm{~g}$ and that BP Psc is a giant star $300 \mathrm{pc}$ distant from the Earth (Z08). For an assumed distance of $80 \mathrm{pc}$, appropriate if BP Psc were a T Tauri star, the mass per grain species is reduced by a factor of $\sim 15$.

${ }^{c}$ Crystalline grains (see Section 3.2).

d Amorphous grains (see Section 3.2).

${ }^{\mathrm{e}}$ We do not consider amorphous olivine as significantly detected in the final $\chi^{2}$ model fit.

${ }^{\mathrm{f}}$ This mass is from Z08 where they computed the disk dust mass assuming half of the $880 \mu \mathrm{m}$ detected flux toward BP Psc comes from a region of the disk having $T_{\text {dust }}=36 \mathrm{~K}$ (fits to the IRS spectrum do not require that an additional $\sim 36 \mathrm{~K}$ dust region exists; Figure 4 of Malfait et al. 1998 shows how the $33 \mu \mathrm{m}$ forsterite feature is sensitive to cool material). If there is no $36 \mathrm{~K}$ dust, then the mass listed for the continuum component could be a few times smaller.

is required despite its low mass fraction and its inclusion in the fit results in a significantly reduced final $\chi^{2}(\sim 84$ if included and $\sim 110$ if not; see Section 7 of Gielen et al. 2008 for how we calculate $\chi^{2}$ ). From Table 1 it is clear that the crystallinity fraction of the small dust grains is high, $\sim 96 \%$, with the majority of this coming from forsterite and enstatite.

The sub-micron sized dust grains orbiting BP Psc, in the case that it is a first-ascent giant star, would be radiatively blown out without a spatially coincident gas disk (this would not be the case if BP Psc were a T Tauri star). To illustrate this, we assume BP Psc has a luminosity of $100 L_{\odot}$ and a mass of $1.8 M_{\odot}$. With Equation (1) from Chen et al. (2006), and assuming grain densities of 3.3 and $2.3 \mathrm{~g} \mathrm{~cm}^{-3}$ for silicates and $\mathrm{SiO}_{2}$ respectively, we estimate that grains smaller than $\sim 10 \mu \mathrm{m}$ in size will be radiatively blown out of the BP Psc system in the absence of gaseous material.

\subsubsection{Unmodeled Features}

Identification of the $\sim 6.5 \mu \mathrm{m}$ absorption/emission complex in BP Psc's IRS spectrum (see Figure 1) is complicated by its weak signature. It is possible that this feature arises from the $v_{2}$ vibrational band of water (see e.g., Woodward et al. 2007). This particular fundamental of gas-phase water presents rovibrational emission features near $6.5 \mu \mathrm{m}$ similar in morphology to the weak features observed at that location in BP Psc's IRS spectrum (compare Figure 1 herein and Figure 2 of Woodward et al. 2007). Since this is not a high fidelity identification, we will refrain from attempting to interpret the presence of water vapor in BP Psc's disk. High-spectral resolution, high signal-to-noise spectra around $6 \mu \mathrm{m}$ will be necessary to reliably identify and characterize this feature. 
There is potentially unmodeled solid-state emission remaining near $20 \mu \mathrm{m}$. If the $20 \mu \mathrm{m}$ emitter is a dust species not already present in the model fit, then it would be unlikely to have other transitions in the $\sim 5-40 \mu \mathrm{m}$ range as the rest of BP Psc's IRS spectrum is reproduced well by the model displayed in Figure 3. A potential carrier of the $20 \mu \mathrm{m}$ emission feature is $\mathrm{FeO}$ nano dust (Zhang et al. 2009 and references therein). $\mathrm{FeO}$ nano dust hosts one solid-state transition near $20 \mu \mathrm{m}$. Temperature controls the width and peak wavelength of the $\mathrm{FeO}$ nano dust feature, with temperatures of $\lesssim 100 \mathrm{~K}$ shifting the feature's peak wavelength to $20.1 \mu \mathrm{m}$ and its FWHM to $2.4 \mu \mathrm{m}$ (Posch et al. 2004 and references therein). Such a peak position and profile (Figure 9 of Posch et al. 2004) could potentially account for the unmodeled emission in BP Psc's IRS spectrum. $\mathrm{FeO}$ nano dust can only exist in environments with low ultraviolet photon density and cool temperatures; ultraviolet photons will dissociate $\mathrm{FeO}$ while high temperatures will prevent $\mathrm{Fe}$ and $\mathrm{O}$ from binding together (Posch et al. 2004). Fe and O orbiting BP Psc at similar locations as the other dust species would be sufficiently cool to bind together while the late spectral type of BP Psc could provide the requisite low ultraviolet photon density to allow the $\mathrm{FeO}$ to stay bound.

\section{COMPARISON TO OTHER CIRCUMSTELLAR DISKS}

In the following sections we compare BP Psc's mid-infrared spectrum to those of other well-studied disk-bearing objects in an effort to better understand the physical environment responsible for BP Psc's disk structure and composition.

\subsection{T Tauri Stars}

Classical T Tauri stars are pre-main sequence, solar-like stars that are still surrounded by substantial amounts of primordial gaseous and dusty material. The IRS indices (see Section 3.1) for these disks are consistent with flat, optically thick, active accretion disks (see e.g., Furlan et al. 2006). BP Psc's IRS indices are strongly discrepant with those for the $\mathrm{T}$ Tauri stars presented in Furlan et al. (2006) and Watson et al. (2009) - where the Taurus median of $n_{6-13}, n_{13-25}$, and $n_{6-25}$ are $-0.82,-0.17$, and -0.48 respectively - suggesting that the structure of BP Psc's disk is significantly different from T Tauri star disk structures (see Section 4.2).

$\mathrm{T}$ Tauri star dusty material shows a variety of compositional characteristics that are at odds or incompatible with what is observed for BP Psc:

1. T Tauri stars sometimes host disks with high crystallinity fractions, but these grains are typically accompanied by significant quantities of amorphous species and are confined to within $\lesssim 10 \mathrm{AU}$ separation from their host stars (Watson et al. 2009). Reasonable age and mass estimates of BP Psc as a classical T Tauri star (see Table 6 of Z08) would require its cool forsterite and enstatite grains to reside at separations $\gtrsim 12 \mathrm{AU}$.

2. If BP Psc were a young T Tauri star, its disk atmosphere would have one of the highest masses in small grains $(\sim 0.1$ lunar masses if located $80 \mathrm{pc}$ from Earth; see notes to Table 1) for a star of its class (e.g., Sargent et al. 2009a, 2009 b, quote a range of mass in small dust grains for the T Tauri stars they studied of $\sim 0.8-700 \times 10^{-4}$ lunar masses).

3. Watson et al. (2009) find in the T Tauri stars they studied an anti-correlation between the strength of the $11 \mu \mathrm{m}$ crystalline olivine feature and the total strength of the entire
$10 \mu \mathrm{m}$ solid-state emission complex. Additionally, Watson et al. (2009) identify a positive correlation between the total strength of the $10 \mu \mathrm{m}$ complex and the magnitude of the $n_{13-25}$ index. These correlations suggest that stronger crystalline emission features, and hence more chemically evolved grain populations, accompany disk settling in T Tauri star disks. Sargent et al. (2009a) found that chemical evolution does not necessarily imply grain growth has occurred, further suggesting that chemical evolution and sedimentation are tied (but Dullemond \& Dominik 2008 show that dust sedimentation alone, without grain growth, cannot reproduce the observed correlations). BP Psc's grains, despite their nearly $100 \%$ crystallinity fraction, are still suspended in the flared disk atmosphere, apparently in contrast with the observed T Tauri star trends.

\subsection{Herbig Ae/Be Stars}

Herbig $\mathrm{Ae} / \mathrm{Be}(\mathrm{HAeBe})$ stars are the intermediate mass counterparts of classical T Tauri stars. HAeBe stars are much hotter and more luminous (median $T_{\text {eff }} \sim 6000-7000 \mathrm{~K}$, $L \sim 10-1000 L_{\odot}$ depending on their progress along premain sequence tracks; see Palla \& Stahler 1993) than lower mass, solar-like $\mathrm{T}$ Tauri stars and hence provide a far different circumstellar environment.

BP Psc's SED is reminiscent of Meeus group I HAeBe stars (see Figure 4 in Meeus et al. 2001). The IRS indices for BP Psc, when plotted in Figure 2 of Acke et al. (2009), lie in the region of infrared excess space describing HAeBe disks having a Meeus group II disk structure (where group II sources have an outer disk which is protected against direct stellar radiation by the puffed-up inner disk rim; see Dullemond \& Dominik 2004 and references therein). This behavior is peculiar, but repeated in a well-studied HAeBe star, HD 100546. This source is given as an exemplar Meeus group I source in Meeus et al. (2001). However, it too resides in the region of Figure 2 of Acke et al. (2009) describing Meeus group II disk structures. Recent interferometric work by Benisty et al. (2010) has confirmed that HD 100546 has a gap in its disk (see Section 6.2). It would seem that gapped disks can provide confusing results when relying on spectral indices alone. Nonetheless, BP Psc's SED, and hence disk structure, is consistent with those found for $\mathrm{HAeBe}$ stars.

The match of BP Psc's disk structure with those of HAeBe stars suggests that their inner disk morphologies are determined from the same physical effect. It is not yet clear what determines this inner radius environment, but the ubiquity of hot excess temperatures of $\sim 1500 \mathrm{~K}$ that fit the near-infrared "bumps" in HAeBe SEDs (thought to arise from puffed-up inner disk rims) suggests that dust sublimation is responsible. This is in contrast to solar mass T Tauri stars, whose inner disk morphologies are determined by magnetospheric accretion (which act on T Tauri disks at $\sim 7 \mathrm{R}_{*}$, close to sublimation temperatures; see Bouvier et al. 2007 and references therein).

Infrared Space Observatory (ISO), IRS, and ground-based mid-infrared spectroscopy of HAeBe stars indicate disks sometimes populated by PAHs and/or significantly evolved dust as evidenced by high mass fractions of larger than sub-micron size, chemically and thermally processed grains (Bouwman et al. 2001; Acke \& van den Ancker 2004; van Boekel et al. 2005; Keller et al. 2008). One HAeBe star, HD 100546, shows dust emission features in its mid-infrared spectrum that are similar to those seen in the IRS spectrum of BP Psc (Figure 2). HD 100546 was shown by Malfait et al. (1998) to have strong contributions to its mid-infrared spectrum from forsterite and PAH emission. 
The grains orbiting HD 100546 are sub-micron sized and originate from two distinct temperature regions (Malfait et al. 1998). More discussion of HD 100546, and its relation to BP Psc, can be found in Section 6.

\subsection{First-ascent Giant Stars}

Three other first-ascent giant stars have well-characterized infrared excess emission. Two of these objects, HD 233517 (a K2 III star; Fekel et al. 1996; Jura 2003; Jura et al. 2006) and HD 100764 (a 4400 K, R-type carbon star; Skinner 1994; Sloan et al. 2007), also have published IRS spectra. The third, TYC 41443292 (an F2-type star; Melis et al. 2009), has existing IRS spectroscopy that will be mentioned here (a more detailed description of TYC 4144329 2's IRS spectrum will appear in C. Melis et al. 2011, in preparation). The infrared excess emission for these three objects is modeled as arising from a Keplerian disk of dusty material that orbits the central giant star. In the case of HD 100764 and TYC 4144329 2, near-infrared excess emission is present indicating dust as hot as $\sim 1500 \mathrm{~K}$ (Skinner 1994; Melis et al. 2009). HD 233517 exhibits infrared excess emission indicating a flared, cool dust disk $\left(T_{\text {dust }} \sim 70 \mathrm{~K}\right)$ orbiting at $\sim 45$ AU separation from the giant star (Jura 2003).

IRS spectroscopy of all three of the above mentioned giant stars indicates that they are orbited by PAHs and small, amorphous, silicate dust grains (Jura et al. 2006; Sloan et al. 2007, C. Melis et al. 2011, in preparation). It is interesting to note that all three of these giant stars host obvious PAH features while BP Psc does not (see Figure 1; although the $\sim 6.5 \mu \mathrm{m}$ feature in BP Psc's IRS spectrum remains unidentified, it is unlikely to be the characteristic $6.2 \mu \mathrm{m}$ PAH transition).

\subsection{Post-AGB Binaries}

With the IRS and TIMMI2 mid-infrared spectrographs, Gielen et al. (2008) performed a comprehensive study of the circumstellar material in Keplerian orbits around post-asymptotic giant branch (post-AGB) binaries having a median stellar effective temperature of $\sim 6000 \mathrm{~K}$ and assumed luminosities in the range of $\sim 5000 \pm 2000 L_{\odot}$. The disks around post-AGB binary systems are well modeled as passive, irradiated disks (Deroo et al. 2007a, 2007b). Some of the post-AGB binary IRS spectra presented in Gielen et al. (2008) have similar slopes as the BP Psc IRS spectrum, indicating that they could have similar structure.

Gielen et al. (2008) found that the stable disks orbiting postAGB binaries host populations of hot and cold amorphous and crystalline grains. Grains in post-AGB binary dust disks show evidence for strong processing, both in the form of grain growth beyond sub-micron sizes and crystallization. The physical process by which disks form in post-AGB binary systems is not well understood, but it is expected that interactions between the evolved star and its companion play a necessary role (Deroo et al. 2007b).

Similar to our discussion of radiative blow out for grains orbiting BP Psc, it can be shown that post-AGB binaries must also have significant quantities of gas spatially coincident with their dusty material. Since the minimum grain size that can orbit a star scales linearly with luminosity, and since post-AGB binary systems have similar masses as first-ascent giants, we estimate that the minimum size grains that can orbit a post-AGB binary in the absence of gas would be $\gtrsim 100 \mu \mathrm{m}$ in size.

\section{IMPLICATIONS FOR THE EVOLUTIONARY STATE OF THE BP PSC SYSTEM}

Z08 established through various methods that BP Psc has the effective temperature of a G8-K0 star and likely has the luminosity of a first-ascent red giant star. We seek to test the luminosity result under the assumption that the effective temperature is correct.

Comparison of BP Psc's dust mineralogy to those of other classes of disk-bearing objects does not provide any significant insight into BP Psc's evolutionary status. Although there are discrepancies with what is observed for classical T Tauri stars, there are also discrepancies with the constituents observed in disks around first-ascent giant stars; for example, other giant stars exhibit detectable PAH emission whereas BP Psc does not. BP Psc, with an accretion rate of $10^{-8} M_{\odot} \mathrm{yr}^{-1}$ (Z08), almost certainly emits sufficient ultraviolet radiation to excite PAH emission; especially if late-type giant stars like HD 233517 and HD 100764, who do not have any obvious accretion, can excite PAH grains. The absence of obvious PAH emission suggests that BP Psc lacks abundant PAH molecules (perhaps the effect of carbon-poor disk material) and/or that the strong near-infrared continuum emission from BP Psc's inner disk rim veils PAH emission features (e.g., Geers et al. 2006). In terms of the presence of a crystalline dust population and lack of PAHs, BP Psc's dust characteristics most resemble those of post-AGB binaries (it is noted that Z08 demonstrated that BP Psc cannot have a luminosity as large as that of an AGB star). BP Psc's dust differs from the objects studied by Gielen et al. (2008) as the majority of BP Psc's grains are sub-micron sized and $\operatorname{cool}\left(T_{\text {dust }}<300 \mathrm{~K}\right)$ while the majority of post-AGB binary stars exhibit hot grains $\left(T_{\text {dust }} \geq 300 \mathrm{~K}\right.$ which results in strong emission features in the $\sim 10 \mu \mathrm{m}$ region) with sizes larger than $\sim 1 \mu \mathrm{m}$.

As discussed in Sections 3 and 4.2, BP Psc's disk has striking structural similarities to disks around $\mathrm{HAeBe}$ stars; in particular, the necessity for the puffed-up inner disk rim that is characteristic of HAeBe disks. This result suggests that $\mathrm{BP}$ Psc, if a classical T Tauri star, lacks strong magnetic fields that effectively funnel material onto the star and prevent the buildup of a substantial inner disk rim. It is hard to imagine BP Psc as a classical T Tauri star without strong magnetic activity (e.g., Preibisch \& Feigelson 2005). Measuring Zeeman splitting of BP Psc's atmospheric absorption lines could provide concrete evidence against the system being pre-main sequence should the lack of a strong magnetic field be verified (Kastner et al. 2010 favor a scenario where BP Psc's X-ray emission is generated through either coronal processes or star-disk interactions, both of which require BP Psc to be magnetically active).

\section{BP PSC'S DISK ORIGIN AND EVOLUTION}

Analysis of the IRS data suggests that BP Psc is more likely an evolved star than a $T$ Tauri star, consistent with the results obtained by Z08 and Kastner et al. (2008, 2010). How did this giant star come to be orbited by so much dusty and gaseous material? Z08 postulate that BP Psc, if an evolved star, could have been rebirthed into its young star-like dusty and gaseous environment by consuming a short-orbital period companion at the onset of its post main-sequence evolution. Such a disk formation mechanism is also favored for the dusty first-ascent giants TYC 41443292 (Melis et al. 2009) and HD 233517 (Jura 2003). Unfortunately, the literature is lacking binary engulfment models that produce results consistent with observed outcomes - giant stars bearing disks in excess of their 
stellar radius and no evidence for the hypothetical consumed companion. The model that provides the most reasonable match to the final observed outcome is outlined in Shu et al. (1979). Their analytical model entails interaction between an evolving star and its short-orbital period, less massive secondary that causes matter to leave the system through the L2 Lagrangian point in a spiral pattern. Eventually the spiral outflow of material will return to strike itself resulting in the formation of a stable disk of material that accretes onto the central giant star and whose maximum size is governed by conservation of angular momentum.

\subsection{Does BP Psc's Dust Composition Result from Binary Engulfment?}

According to the Shu et al. (1979) model, material lost from binary interaction will initially be close to the host giant star and thus would be subjected to the intense giant star radiation field. Once this material passes beyond the sublimation radius it can begin to coalesce into dust grains. These dust grains should be efficiently annealed with the heat from the nearby luminous star, resulting in a high fraction of dust being crystalline. In their study of the $10 \mu \mathrm{m}$ emission feature of HAeBe stars, Bouwman et al. (2001) found that with increasing abundances of forsterite one expects to see larger mass fractions of crystalline $\mathrm{SiO}_{2}$. Such a result is indicative of the thermal annealing process which transforms amorphous into crystalline grains with $\mathrm{SiO}_{2}$ as a by-product. That we see $\mathrm{SiO}_{2}$ in the material surrounding BP Psc lends some support to a disk formation model similar to that presented by Shu et al. (1979). The orbital semi-major axis of this crystalline material will continue to expand until the disk reaches its maximum size. Thus, highly crystalline material would be transported to the cooler, outer regions of the disk where it is observed.

There are some problems with the above grain evolution scenario. One is that other first-ascent giants with dusty material thought to originate in a manner similar to BP Psc's do not show evidence for highly crystalline material. A second problem is that the polymorph of $\mathrm{SiO}_{2}$ most likely present in BP Psc's IRS spectrum, cristobalite, must be cooled very quickly after being annealed or it will revert to lower temperature polymorphs that do not exhibit the characteristic $16 \mu \mathrm{m}$ emission feature (Sargent et al. 2009b). It is difficult to imagine that the disk excretion could be slow enough to efficiently anneal $\mathrm{SiO}_{2}$ into a cristobalite polymorph and at the same time rapid enough to transport this cristobalite to cooler regions before it can revert. More detailed modeling and simulations of disk formation through engulfment could further explore these issues.

\subsection{A Planetary Model to Account for BP Psc's Observed Disk SED and Composition}

It seems unlikely that the composition of the dust orbiting BP Psc is linked to its disk formation mechanism due to the problems discussed above. Instead, the dust mineralogy could be the result of evolutionary processes which occur in protoplanetary disks and are understood to be the precursors to (or indicators of) planet/planetesimal formation. Under such an assumption the IRS results could provide insight into whether or not new planetesimal formation is occurring in BP Psc's disk. As discussed above, the presence of cristobalite would require an event that heated the disk to temperatures of $\sim 1000$ $\mathrm{K}$ and then allowed it to cool rapidly. Such an event likely took place in BP Psc's outer disk where the disk equilibrium temperature is sufficiently low to prevent the cristobalite from reverting (Sargent et al. 2009b). As discussed in Sargent et al. (2009b), disk-lightning or nebular shock scenarios can explain the presence of cool, highly crystalline material (especially cristobalite). However, they do not explain why so much of this material would be in BP Psc's flared disk atmosphere (lightning and shocks are expected to mainly act in the disk interior; Pilipp et al. 1998; Harker \& Desch 2002), especially when one considers that grain processing should be linked with grain growth and sedimentation (e.g., Natta et al. 2007; Watson et al. 2009 and references therein).

Potential solutions to these problems come from comparing BP Psc to HD 100546. Bouwman et al. (2003) suggest that HD 100546 - which has an SED (Figure 2) and crystalline silicate emission similar to those of BP Psc (with the exception of the presence of $\mathrm{SiO}_{2}$ ) - has a proto-gas giant planet carving a gap within its disk. Such a gap naturally explains the enhanced mid- to far-infrared excess emission relative to the near-infrared excess emission in HD 100546 when compared to other HAeBe stars as the far side of the gap would be directly irradiated by the star. This direct stellar irradiation would produce a vertically extended wall in the disk, allowing it to intercept a large fraction of stellar light which would then be reradiated as blackbody emission having the characteristic temperature for the disk gap location ( $200 \mathrm{~K}$ for HD 100546; see Figure 2). Interferometric observations of HD 100546 by Benisty et al. (2010) confirm that HD 100546 does indeed have a gap within its disk. Recent optical and ultraviolet spectroscopic studies of HD 100546 further support the interpretation that this star has a giant planet orbiting within the gap in its disk (Grady et al. 2005; Acke \& van den Ancker 2006). BP Psc and HD 100546 have similar SEDs (Figure 2) and thus could potentially have similar disk structure (it is noted that BP Psc's disk inclination angle $\sim 75^{\circ}$ is closer to edge-on than HD 100546's $\sim 51^{\circ}$ and that edge-on disks without gaps around young solar-mass stars are known to have "double-hump" SEDs; Grady et al. 2001; Wood et al. 2002). If this were the case, it would be reasonable to surmise that the same mechanism responsible for HD 100546's disk structure (a gap-opening giant planet) operates also in BP Psc's disk. Interferometric observations of BP Psc as well as detailed modeling of its resolved disk images and SED are necessary to confirm such a gapped disk model. Any such giant planet orbiting BP Psc would have an orbital semi-major axis of $\sim 4 \mathrm{AU}$ (assuming BP Psc has a luminosity of $\sim 100 L_{\odot}$ ). We note that theoretical works predict that any such object have a mass that is $<10 M_{\text {Jup }}$ as more massive bodies would restrict the flow of material toward the star resulting in a depleted inner disk (Lubow et al. 1999). Such depletion would not be compatible with observations that indicate large quantities of gaseous and dusty material in BP Psc's inner disk (Z08). It is not clear whether any such giant planet would be from BP Psc's original planetary system or if it would be a protoplanet forming from the material in BP Psc's giant star disk.

Two mechanisms to explain the observed dust mineralogy are discussed in the following subsections. Both rely on the existence of a giant planet opening a gap within BP Psc's disk.

\subsubsection{Colliding Planetesimals}

A giant planet orbiting within BP Psc's disk could stir up rocky bodies that also orbit in the disk gap. These rocky bodies could be remnant planetary building material left over from BP Psc's first planetary system that orbit at similar stellar separations as the giant planet (like Jupiter's trojan asteroids) or they 
could have formed in situ in BP Psc's revived giant star disk. Following Bouwman et al. (2003), we expect ensuing collisions between these stirred planetesimals to release previously generated crystalline material. This collisional residue is then blown out of the disk gap by various processes (see Section 6.2.2) and eventually lands in the disk atmosphere where it is observed.

If the colliding rocky objects originated from BP Psc's giant star disk material, then they likely formed through similar pathways as planetesimals forming around young stars. Another common indicator of planetesimal formation in young stars is grain growth to millimeter-centimeter sizes (e.g., Natta et al. 2007 and references therein). Is there any evidence for grain growth in the disk around BP Psc? Z08 report $880 \mu \mathrm{m}$ measurements of BP Psc and interpret the continuum flux as originating from a population of cooler or potentially larger grains. The only other longer wavelength data point that exists for BP Psc that could be used to constrain grain growth is an upper limit reported at $1 \mathrm{~mm}$ (Z08). This upper limit, however, does not allow one to place any stringent limits on the maximum grain size within BP Psc's dusty disk. Limits on the growth of grains in the disk around BP Psc will require more constraining observations at wavelengths longer than $880 \mu \mathrm{m}$.

There are some pitfalls in this proposed dust enrichment model. If the colliding planetesimals are left over from BP Psc's initial planetary system, then there must be a large population of trojan-like bodies whose orbits evolve to bring them closer to the giant planet. In theory, interactions between such rocky bodies and BP Psc's gaseous disk could alter the rocky bodies' orbits and bring a sufficient number of them close enough to the giant planet to experience enhanced collisional activity (the likes of which must produce $\sim 1$ lunar mass of small crystalline grains; see Table 1). Of course, if the planetesimals are instead being formed in situ in the giant star disk, then this problem disappears. Another problem is that Bouwman et al. (2003) do not provide a convincing argument as to why one should expect isolated regions of nearly $100 \%$ crystalline dust in the disk atmosphere when the colliding bodies that produce the dust are more compositionally diverse (see the middle panel of Figures 5 and 10 and Table 3 in Bouwman et al. 2003).

\subsubsection{Giant Planet Disk Shocking}

Another possibility is that a giant planet generates shocks in the disk that sufficiently increase the disk temperature to anneal amorphous grains. Many theoretical works exist that predict shocks in the edges of a disk gap as a result of tidal interactions between a (gap-opening) giant planet and the disk (e.g., Takeuchi et al. 1996; Lubow et al. 1999; Rafikov 2002). These shocks could act to heat small, amorphous grains to sufficient temperatures such that thermal annealing into crystalline species would result (Harker \& Desch 2002). The most interesting aspect of this model is the dust mineralogy it predicts. Annealing Mg-rich amorphous grains results in the generation of forsterite and $\mathrm{SiO}_{2}$ as a by-product, while reaction of forsterite and $\mathrm{SiO}_{2}$ results in the production of enstatite (Bouwman et al. 2001 and references therein). Thus, giant planet-induced shocks in the disk are capable of reproducing the observed small grain mineralogy. Furthermore, shocks are the favored production method for cristobalite (Sargent et al. 2009 b), the most likely polymorph of $\mathrm{SiO}_{2}$ observed in $\mathrm{BP}$ Psc's disk.

After shocks produce the crystalline material in BP Psc's disk, the processed dust must be relocated from the disk midplane into the disk atmosphere where it is observed. Assuming shock-generated crystalline material enters the disk gap, it may then be orbitally perturbed (both radially and vertically) by the giant planet such that it may be directly irradiated by stellar light and driven out of the disk mid-plane by radiation pressure. The flared disk geometry will allow these liberated grains to be re-captured by the disk, although at larger separations than where they originated. Thus, the disk atmosphere will become polluted with highly crystalline material as is observed for BP Psc.

This is our preferred model as it naturally accounts for both BP Psc's SED and mineralogy.

\section{CONCLUSIONS}

We have obtained and analyzed Spitzer IRS spectra for the enigmatic dust and gas-enshrouded star BP Psc. Disk structure analysis suggests that BP Psc's disk is unlike any classical $\mathrm{T}$ Tauri star disk, but is instead similar to disks around Herbig $\mathrm{Ae} / \mathrm{Be}$ stars. Such a result suggests that BP Psc is itself as luminous as these pre-main sequence, intermediate mass stars. The suite of evidence presented in Z08 requires that BP Psc-if it is indeed as luminous as a typical HAeBe star-be a firstascent giant star. The match of BP Psc's SED with that of the HAeBe star HD 100546 suggests that BP Psc's disk contains a gap. Evidence for a giant planet as the generator of the gap in HD 100546's disk suggests that BP Psc could also have a giant planet orbiting within its disk. Dust model fits to the IRS spectrum show that BP Psc's flared disk atmosphere contains chemically and thermally processed, cool, small dust grains. Our preferred model to explain the location and mineralogy of these grains relies on a giant planet orbiting BP Psc. Disk shocks that result from disk-planet interaction generate the highly crystalline dust which is subsequently removed from the disk mid-plane and relocated into the disk atmosphere where it is observed. We note, as suggested by Bouwman et al. (2003), that this highly crystalline dust could be the seed material that forms cometary bodies like Hale-Bopp. If this is the case, and if BP Psc is indeed a first-ascent giant star, then we could be witnessing the initial formation stages of a second generation of cometary bodies around BP Psc.

C.M. acknowledges support from the Spitzer Visiting Graduate Student Program and from a LLNL Minigrant to UCLA. This work was supported in part by the National Science Foundation under award No. AST-1003318. We thank the IRS GTO team for carrying out these observations and Michiel Min for providing us with absorption coefficients for the various dust species. We also thank Carey Lisse for useful discussion and providing absorption coefficients for cristobalite. We thank an anonymous referee for a thorough report that helped improve this paper. This research was supported in part by NASA grants to UCLA and the University of Georgia.

Facilities: Spitzer (IRS, MIPS)

\section{REFERENCES}

Acke, B., Min, M., van den Ancker, M. E., Bouwman, J., Ochsendorf, B., Juhasz, A., \& Waters, L. B. F. M. 2009, A\&A, 502, L17

Acke, B., \& van den Ancker, M. E. 2004, A\&A, 426, 151

Acke, B., \& van den Ancker, M. E. 2006, A\&A, 449, 267

Baraffe, I., Chabrier, G., Allard, F., \& Hauschildt, P. H. 1998, A\&A, 337, 403

Benisty, M., Tatulli, E., Ménard, F., \& Swain, M. R. 2010, A\&A, 511, A75

Bouvier, J., Alencar, S. H. P., Harries, T. J., Johns-Krull, C. M., \& Romanova, M. M. 2007, in Protostars and Planets V, ed. B. Reipurth, D. Jewitt, \& K. Keil (Tucson, AZ: Univ. Arizona Press), 479 
Bouwman, J., de Koter, A., Dominik, C., \& Waters, L. B. F. M. 2003, A\&A, 401, 577

Bouwman, J., Meeus, G., de Koter, A., Hony, S., Dominik, C., \& Waters, L. B. F. M. 2001, A\&A, 375, 950

Chen, C. H., et al. 2006, ApJS, 166, 351

Deroo, P., Acke, B., Verhoelst, T., Dominik, C., Tatulli, E., \& van Winckel, H. 2007a, A\&A, 474, L45

Deroo, P., van Winckel, H., Verhoelst, T., Min, M., Reyniers, M., \& Waters, L. B. F. M. 2007b, A\&A, 467, 1093

Draine, B. T. 1988, ApJ, 333, 848

Dullemond, C. P., \& Dominik, C. 2004, A\&A, 417, 159

Dullemond, C. P., \& Dominik, C. 2008, A\&A, 487, 205

Fekel, F. C., Webb, R. A., White, R. J., \& Zuckerman, B. 1996, ApJ, 462, L95

Furlan, E., et al. 2006, ApJS, 165, 568

Geers, V. C., et al. 2006, A\&A, 459, 545

Gervais, F., \& Piriou, B. 1975, Phys. Rev. B, 11, 3944

Gielen, C., van Winckel, H., Min, M., Waters, L. B. F. M., \& Lloyd Evans, T. 2008, A\&A, 490, 725

Grady, C. A., Woodgate, B., Heap, S. R., Bowers, C., Nuth, III, J. A., Herczeg, G. J., \& Hill, H. G. M. 2005, ApJ, 620, 470

Grady, C. A., et al. 2001, AJ, 122, 3326

Harker, D. E., \& Desch, S. J. 2002, ApJ, 565, L109

Hauschildt, P. H., Allard, F., \& Baron, E. 1999, ApJ, 512, 377

Houck, J. R., et al. 2004, ApJS, 154, 18

Jura, M. 2003, ApJ, 582, 1032

Jura, M., et al. 2006, ApJ, 637, L45

Kastner, J. H., Zuckerman, B., \& Forveille, T. 2008, A\&A, 486, 239

Kastner, J. H., et al. 2010, ApJ, 719, L65

Keller, L. D., et al. 2008, ApJ, 684, 411

Lisse, C. M., et al. 2006, Science, 313, 635

Lubow, S. H., Seibert, M., \& Artymowicz, P. 1999, ApJ, 526, 1001

Malfait, K., Waelkens, C., Waters, L. B. F. M., Vandenbussche, B., Huygen, E., \& de Graauw, M. S. 1998, A\&A, 332, L25
Meeus, G., Waters, L. B. F. M., Bouwman, J., van den Ancker, M. E., Waelkens, C., \& Malfait, K. 2001, A\&A, 365, 476

Melis, C. A. 2009, PhD thesis, Univ. California, Los Angeles

Melis, C., Zuckerman, B., Song, I., Rhee, J. H., \& Metchev, S. 2009, ApJ, 696, 1964

Min, M., Waters, L. B. F. M., de Koter, A., Hovenier, J. W., Keller, L. P., \& Markwick-Kemper, F. 2007, A\&A, 462, 667

Natta, A., Testi, L., Calvet, N., Henning, T., Waters, R., \& Wilner, D. 2007, in Protostars and Planets V, ed. B. Reipurth, D. Jewitt, \& K. Keil (Tucson, AZ: Univ. Arizona Press), 767

Palla, F., \& Stahler, S. W. 1993, ApJ, 418, 414

Pilipp, W., Hartquist, T. W., Morfill, G. E., \& Levy, E. H. 1998, A\&A, 331, 121

Posch, T., Mutschke, H., \& Andersen, A. 2004, ApJ, 616, 1167

Preibisch, T., \& Feigelson, E. D. 2005, ApJS, 160, 390

Rafikov, R. R. 2002, ApJ, 572, 566

Rieke, G. H., et al. 2004, ApJS, 154, 25

Sargent, B. A., et al. 2009a, ApJS, 182, 477

Sargent, B. A., et al. 2009b, ApJ, 690, 1193

Shkuratov, Y. G., \& Grynko, Y. S. 2005, Icarus, 173, 16

Shu, F. H., Anderson, L., \& Lubow, S. H. 1979, ApJ, 229, 223

Skinner, C. J. 1994, MNRAS, 271, 300

Sloan, G. C., et al. 2007, ApJ, 664, 1144

Takeuchi, T., Miyama, S. M., \& Lin, D. N. C. 1996, ApJ, 460, 832

van Boekel, R., Min, M., Waters, L. B. F. M., de Koter, A., Dominik, C., van den Ancker, M. E., \& Bouwman, J. 2005, A\&A, 437, 189

Watson, D. M., et al. 2009, ApJS, 180, 84

Werner, M. W., et al. 2004, ApJS, 154, 1

Wood, K., Lada, C. J., Bjorkman, J. E., Kenyon, S. J., Whitney, B., \& Wolff, M. J. 2002, ApJ, 567, 1183

Woodward, C. E., Kelley, M. S., Bockelée-Morvan, D., \& Gehrz, R. D. 2007, ApJ, 671, 1065

Zhang, K., Jiang, B. W., \& Li, A. 2009, MNRAS, 396, 1247

Zuckerman, B., et al. 2008, ApJ, 683, 1085 (Z08) 